\title{
Does the Loudness Dependence of Auditory Evoked Potential Predict Response to Selective Serotonin Reuptake Inhibitors?: A Meta-analysis
}

\author{
Sunkyung Yoon', Yourim Kim², Seung-Hwan Lee ${ }^{2,3}$ \\ ${ }^{1}$ Department of Psychology, University of South Florida, Tampa, FL, USA, ${ }^{2}$ Clinical Emotion and Cognition Research Laboratory, ${ }^{3}$ Department \\ of Psychiatry, Inje University Ilsan Paik Hospital, Goyang, Korea
}

\begin{abstract}
Objective: Loudness of dependence of the auditory evoked potential (LDAEP) is an electroencephalogram-based measure that represents amplitude changes of auditory evoked potentials in primary auditory cortex. Several narrative reviews argued that pre-treatment LDAEP values predict responses to selective serotonin reuptake inhibitors (SSRIs). This study aims to quantify the overall relationship between baseline LDAEP values and treatment response to SSRIs in patients with depression and generalized anxiety disorders, evidenced by clinical symptoms reductions, across multiple studies. Methods: In our meta-analysis, seven articles with a total sample of 241 patients were included.

Results: Our results showed that stronger baseline LDAEP values predicted favorable response to SSRIs for depression and anxiety, with a moderate effect size.

Conclusion: The current results support the idea that LDAEP is a promising biomarker for SSRIs treatment prediction in patients with depression and generalized anxiety disorder.
\end{abstract}

KEY WORDS: Electroencephalogram; Prediction; LDAEP; Selective serotonin reuptake inhibitors; Major depressive disorder; Generalized anxiety disorder.

\section{INTRODUCTION}

Many previous studies have demonstrated that serotonin (5-hydroxytryptamine, 5-HT) and its dysfunction play an important role in the development and treatment of various psychiatric disorders, especially depression and anxiety disorders [1-3]. Selective serotonin reuptake inhibitors (SSRIs), which selectively target increasing 5-HT levels in the brain by inhibiting its reuptake, have been considered as the first line treatment for depression and anxiety over the past decades $[4,5]$. Despite abundant evidence on the significant link between 5-HT dysfunction and depression/anxiety, treatment response to SSRIs (e.g., reduction in clinical symptoms) are not always favorable

Received: April 27, 2020 / Revised: June 5, 2020

Accepted: June 16, 2020

Address for correspondence: Seung-Hwan Lee

Department of Psychiatry, Inje University Ilsan Paik Hospital, 170 Juhwa-ro, Ilsanseo-gu, Goyang 10380, Korea

E-mail: Ishpss@paik.ac.kr

ORCID: https://orcid.org/0000-0003-0305-3709
[6]. To increase the efficacy of treatments and treatment selection, it is crucial to identify a marker(s) that reliably predicts response to SSRIs and can be readily measured in clinical settings.

This paper focuses on the loudness of dependence of the auditory evoked potential (LDAEP) as a potential biological marker to predict response to SSRIs for patients with depression and anxiety disorders. LDAEP is an electroencephalogram (EEG)-based measure that represents amplitude changes of auditory evoked potentials in primary auditory cortex, $\mathrm{N} 1$ and $\mathrm{P} 2$, in response to auditory stimuli with varying intensities. Initially, LDAEP has received attention due to empirical evidence showing that the changes in N1/P2 amplitudes with varying stimuli intensities (N1/P2 LDAEP) have an inverse association with 5-HT functions [7]: the stronger N1/P2 LDAEP is related to lower levels of 5-HT functions. However, a comprehensive review on the validity and utility of LDAEP [8] concluded that previous results are inconsistent on its validity as a marker for 5-HT neurotransmission.

(c) This is an Open-Access article distributed under the terms of the Creative Commons Attribution Non-Commercial License (http://creativecommons.org/licenses/by-nc/4.0) which permits unrestricted non-commercial use, distribution, and reproduction in any medium, provided the original work is properly cited. 
Several narrative reviews indicated that LDAEP appears to have a potential predictability of response to SSRIs [8-11]. For example, O'Neil and colleagues (2008), who challenged the idea of LDAEP as a reliably marker for 5-HT function, highlighted its potential utility as a treatment predictor: "In the meantime, the most promising utility of the LDAEP is as a clinical marker of initial antidepressant treatment response" (p. 366) [8]. LDAEP can be a compelling clinical measure because it is not invasive and relatively easy to administer in terms of cost and required resources, which makes it feasible to become a routine procedure in clinical practice. This paper aims to investigate whether pre-treatment LDAEP values predict response to SSRIs for depression or anxiety disorders across multiple studies and samples, using a meta-analysis.

We believe a meta-analysis is needed because no study has yet quantified the overall predictability of baseline LDAEP to response to SSRIs across multiple studies. Although narrative reviews summarized that stronger LDAEP predicts favorable response to SSRIs [8-11], the magnitude of the relationship remains unknown. A metaanalysis will be able to elucidate both direction and magnitude of the relationship. Further, previous studies on
LDAEP and treatment response tend to have a small sample size, which was noted as a limitation $[12,13]$.

The current meta-analysis tested the hypothesis that stronger pre-treatment N1/P2 LDAEP values predict better response to SSRIs for depression and anxiety disorders. Favorable response was defined as greater symptoms reduction at the post-treatment, relative to the pre-treatment, after SSRIs treatment.

\section{METHODS}

\section{Search Strategy}

A systematic search was performed with articles published by March 2020, in PubMed, PsycINFO, and ProQuest dissertations \& Theses Global. The search was complemented by Google Scholar. To be inclusive in our search, we used broad keywords searched within abstracts: "loudness dependence of auditory evoked potentials (LDAEP)" OR "intensity dependence of the auditory evoked potentials (IDAEP)". The flow chart of the process is presented in Figure 1.

\section{Eligibility Criteria}

We included articles that 1) were written in English, 2)

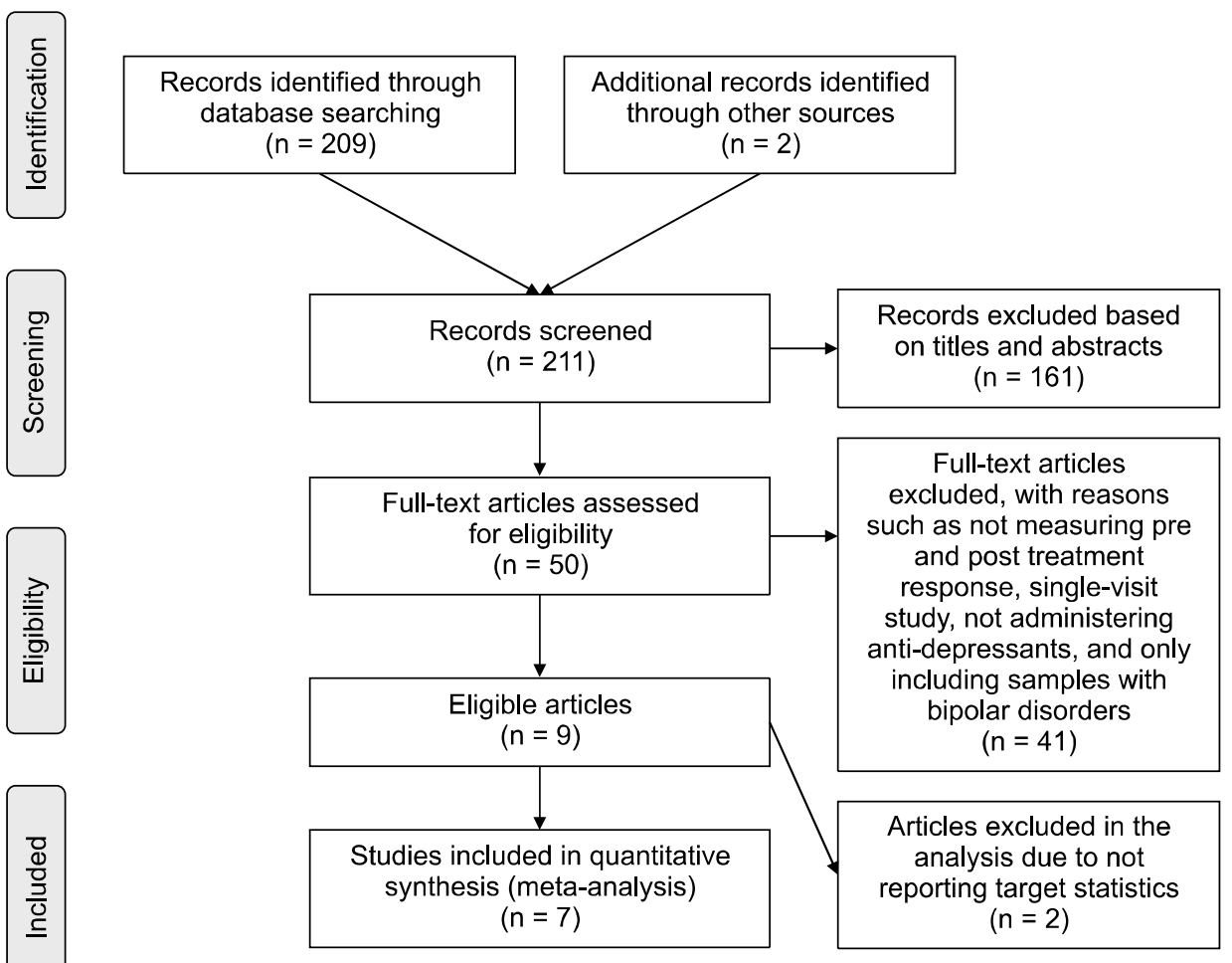

Fig. 1. Flow chart of literature review. 
conducted in human, 3) measured N1/P2 LDAEP, and 4) administered SSRIs as a treatment, and 5) assessed treatment response by measuring symptom changes between pre- and post-treatment. We did not have specific inclusion criteria for a primary diagnosis; however, articles were excluded when they included participants with a primary diagnosis of current bipolar disorders or any psychotic disorders.

\section{Coding Procedure}

We coded following variables: (1) effect size, (2) sample size, (3) sex ratio (female ratio, \%), (4) patient type (1 = outpatient, 2 = inpatient), and (5) treatment duration. The first and second author searched literature using the same keywords and coded the included studies. Seven out of nine $(77 \%)$ eligible articles were found by both authors. The Kappa coefficient for the recorded categorical variable (patient type) was 0.70 between the first and the second authors. For the continuous variables, intra-class correlations ranged from 0.97 (female ratio) to 1 (sample size) between the first and second authors. Disagreement between raters was resolved via discussion. These results showed adequate inter-rater agreement [14].

\section{Statistical Methods}

There was no single effect size most frequently used among the included articles. We chose correlation coefficients $r$ because three articles reported correlations between pre-treatment LDAEP values and symptom reductions after antidepressants, and it is easy to interpret. Other statistics (i.e., $z$ values of non-parametric tests, contingency table of responders vs. non-responders based on LDAEP values, the mean baseline LDAEP values between responders vs. non-responders) were converted to $r$. Correlation coefficients were transformed using the Fisher's $z$ transformation and analyzed to calculate the average effect size, and the $z$ values were converted back to correlation scores for presentation. The magnitude of effect size “ $r$ " was interpreted based on Cohen [15]: $r=0.10$ as small, 0.30 as medium, and 0.50 and above as large.

Using the metaphor package in R software [16], a random-effects model was conducted with restricted maximum-likelihood (REML). We computed 95\% prediction intervals to estimate the possible range of true effects and calculated the $95 \%$ confidence intervals $(95 \% \mathrm{Cl})$ for the overall mean effect. $Q$ test was used for testing hetero- geneity, and visual inspection of the funnel plot and Egger's regression test were performed to evaluate publication bias. When potential publication bias was detected, we used the trim and fill method to adjust the bias.

\section{RESULTS}

\section{Characteristics of Included Studies}

Seven articles were included with a total sample of 241 (mean sample size $=34.42$, range $=11-100$ ). Two articles $[17,18]$ reported results on citalopram (SSRI) and reboxetine (norepinephrine reuptake inhibitor, NRI), respectively. For the current meta-analysis, we included only the results of patients who were treated with citalopram. One article [18] did not report sex and age specifically for participants who received the SSRIs treatment. The mean age across studies was 46.74 , and the mean female participant ratio was approximately $64 \%$. All but one study examined patients who met the diagnosis of current major depressive disorder or persistent depressive disorder (i.e., dysthymia), according to the Diagnostic and Statistical Manual of Mental Disorders, 3rd or 4th edition and/or International Classification of Diseases, 10th revision (ICD-10), whereas Park et al. [13]'s participants had current generalized anxiety disorder (GAD). Two studies $[12,13]$ included outpatients while the others had inpatients. All but one study [19] defined treatment response as an equal to and greater than $50 \%$ decrease in symptom scores at the post-treatment, compared to pre-treatment scores. Lee et al. [19], measured treatment response by the percentage of symptom reduction at post-treatment, compared to pre-treatment (i.e., [pre-treatment symptom score - post-treatment score/pre-treatment score] $\times 100$ ).

There was variability in EEG data acquisition and analysis methods among the included studies. The most commonly used assessment and analysis method were as follows: EEG was measured in a sound-attenuated room using headphones and with participants' eyes open. Five tone intensities were used (mostly around 60, 70, 80, 90, $100 \mathrm{~dB}$ levels) at $1,000 \mathrm{~Hz}$ with $40 \mathrm{msec}$ duration and inter-stimulus-interval of 1,800-2,200 msec. N1/P2 was defined and computed as a linear regression slope of $\mathrm{N} 1$ and P2 in the window of 63.5-280 after the stimulus onset, although there was a significant difference in this range among the included studies. Three included studies used dipole source analysis $[17,20,21]$ and the rest four 
Table 1. Characteristics of included articles

\begin{tabular}{|c|c|c|c|c|c|c|c|c|}
\hline Study & Sample & Diagnosis & $\begin{array}{l}\text { Age } \\
(y r)\end{array}$ & $\begin{array}{c}\text { Female } \\
(\%)\end{array}$ & Medication & $\begin{array}{l}\text { Treatment } \\
\text { duration }\end{array}$ & $\begin{array}{l}\text { Symptoms } \\
\text { measure }\end{array}$ & Data acquisition/analysis \\
\hline $\begin{array}{l}\text { Gallinat et al., } \\
2000[20]\end{array}$ & $\begin{array}{c}\text { Inpatients } \\
(\mathrm{n}=29)\end{array}$ & $\begin{array}{l}\text { MDD }(n=28) \\
\text { Dysthymia }(n=1)\end{array}$ & 55.6 & 48 & $\begin{array}{l}\text { Paroxetine } \\
\text { Sertraline } \\
\text { Citalopram }\end{array}$ & 4 weeks & $\begin{array}{l}\text { HAM-D } \\
21 \text { items }\end{array}$ & $\begin{array}{l}\text { Eyes open } \\
32 \text { channels } \\
\text { Tone }=1,000 \mathrm{~Hz} / 40 \mathrm{msec} \\
\mathrm{ISI}=1,800-2,200 \mathrm{msec} \\
5 \text { intensities }(54,64,74,84,94 \mathrm{~dB}) \\
\text { Sampling rate }=256 \mathrm{~Hz} \\
\mathrm{~N} 1 / \mathrm{P} 2=63.5-217 \mathrm{msec} \\
\text { Dipole source analysis }\end{array}$ \\
\hline $\begin{array}{l}\text { Juckel et al., } \\
2007 \text { [17] }\end{array}$ & $\begin{array}{l}\text { Inpatients } \\
(\mathrm{n}=20)\end{array}$ & $\begin{array}{l}\text { MDD without } \\
\text { psychotic features }\end{array}$ & 44.3 & 70 & Citalopram & 4 weeks & HAM-D & $\begin{array}{l}\text { Eyes open } \\
32 \text { channels } \\
\text { Tone }=1,000 \mathrm{~Hz} / 40 \mathrm{msec} \\
\mathrm{ISI}=1,800-2,200 \mathrm{msec} \\
5 \text { intensities }(60,70,80,90,100 \mathrm{~dB}) \\
\text { Sampling rate }=256 \mathrm{~Hz} \\
\mathrm{~N} 1 / \mathrm{P} 2=63.5-217 \mathrm{msec} \\
\text { Dipole source analysis }\end{array}$ \\
\hline $\begin{array}{l}\text { Lee et al., } \\
2005 \text { [19] }\end{array}$ & $\begin{array}{l}\text { Inpatients } \\
\quad(\mathrm{n}=100)\end{array}$ & MDD & 42.9 & 60 & Fluoxetine & 4 weeks & $\begin{array}{l}\text { HAM-D } \\
17 \text { items }\end{array}$ & $\begin{array}{l}\text { Eyes open } \\
\text { Tone }=2,000 \mathrm{~Hz} / 10 \mathrm{msec} \\
\mathrm{ISI}=1,800-2,200 \mathrm{msec} \\
4 \text { intensities }(60,70,80,90 \mathrm{~dB}) \\
\text { Sampling rate }=256 \mathrm{~Hz} \\
\mathrm{~N} 1=65-175 \mathrm{msec} \\
\mathrm{P} 2=120-280 \mathrm{msec} \\
\text { Cortical analysis }\end{array}$ \\
\hline $\begin{array}{l}\text { Lee et al., } \\
2015 \text { [12] }\end{array}$ & $\begin{array}{l}\text { Outpatients } \\
(\mathrm{n}=41)\end{array}$ & MDD & 40.2 & 83 & $\begin{array}{l}\text { Escitalopram } \\
\text { Sertraline } \\
\text { Paroxetine }\end{array}$ & $\begin{array}{l}14.1 \pm 2.1 \\
\text { weeks }^{\mathrm{a}}\end{array}$ & $\mathrm{BDI}$ & $\begin{array}{l}\text { Eyes open } \\
64 \text { channels } \\
\text { Tone }=1,000 \mathrm{~Hz} / 80 \mathrm{msec} \\
\mathrm{ISI}=500-900 \mathrm{msec} \\
5 \text { intensities }(55,65,75,85,95 \mathrm{~dB}) \\
\text { Sampling rate }=1,000 \mathrm{~Hz} \\
\mathrm{~N} 1=80-130 \mathrm{msec} \\
\mathrm{P} 2=130-230 \mathrm{msec} \\
\text { Cortical analysis }\end{array}$ \\
\hline $\begin{array}{l}\text { Mulert et al., } \\
2002[21]\end{array}$ & $\begin{array}{r}\text { Inpatients } \\
(\mathrm{n}=15)\end{array}$ & MDD & 45 & 66 & Citalopram & 4 weeks & $\begin{array}{l}\text { HAM-D } \\
21 \text { items }\end{array}$ & $\begin{array}{l}\text { Eyes open } \\
32 \text { channels } \\
\text { Tone }=1,000 \mathrm{~Hz} / 40 \mathrm{msec} \\
\mathrm{ISI}=1,800-2,200 \mathrm{msec} \\
5 \text { intensities }(60,70,80,90,100 \mathrm{~dB}) \\
\text { Sampling rate }=256 \mathrm{~Hz} \\
\mathrm{~N} 1 / \mathrm{P} 2=60-240 \mathrm{msec} \\
\text { Dipole source analysis }\end{array}$ \\
\hline $\begin{array}{l}\text { Mulert et al., } \\
2007 \text { [18] }\end{array}$ & $\begin{array}{c}\text { Inpatients } \\
(\mathrm{n}=11)\end{array}$ & MDD & & & Citalopram & 4 weeks & $\begin{array}{l}\text { HAM-D } \\
21 \text { items }\end{array}$ & $\begin{array}{l}\text { Eyes closed } \\
32 \text { channels } \\
\text { Tone }=1,000 \mathrm{~Hz} / 40 \mathrm{msec} \\
\mathrm{ISI}=1,800-2,200 \mathrm{msec} \\
5 \text { intensities }(60,70,80,90,100 \mathrm{~dB}) \\
\text { Sampling rate }=250 \mathrm{~Hz} \\
\mathrm{~N} 1 / \mathrm{P} 2=60-240 \mathrm{msec} \\
\text { Cortical analysis }\end{array}$ \\
\hline $\begin{array}{l}\text { Park et al., } \\
2011 \text { [13] }\end{array}$ & $\begin{array}{l}\text { Outpatients } \\
(\mathrm{n}=25)\end{array}$ & GAD & 52.3 & 60 & Escitalopram & 8 weeks & HAM-A & $\begin{array}{l}64 \text { channels } \\
\text { Tone }=1,000 \mathrm{~Hz} / 80 \mathrm{msec} \\
\mathrm{ISI}=500-900 \mathrm{msec} \\
5 \text { intensities }(55,65,75,85,95 \mathrm{~dB}) \\
\text { Sampling rate }=1,000 \mathrm{~Hz} \\
\mathrm{~N} 1=80-130 \mathrm{msec} \\
\mathrm{P} 2=130-230 \mathrm{msec} \\
\text { Cortical analysis }\end{array}$ \\
\hline
\end{tabular}

MDD, major depressive disorder; BDI, beck depression inventory, self-reported; HAM-D, Hamilton scale for depression, clinician-administered; HAM-A, Hamilton scale for anxiety, clinician-administered; ISI, inter stimulus interval.

${ }^{\mathrm{a} M e a n} \pm$ standard deviation. 
studies used cortical analysis. All studies used or included $\mathrm{Cz}$ as a reference channel, and therefore LDAEP values determined at $\mathrm{Cz}$ were used in the current analysis. Detailed characteristics of included articles are presented in Table 1.

\section{Association between LDAEP and Response to Antidepressants}

A random-effects model of the LDAEP and SSRIs treatment response yielded a medium positive correlation $(r=$ $0.33,95 \% \mathrm{Cl}[0.20,0.45], z=4.61, p<0.001,95 \%$ credibility intervals $[C R][0.15,0.49])$. As suggested in previous narrative reviews, our results showed that higher pre-treatment LDAEP predicts better response to antidepressants across studies. The $Q$ test statistics indicated no significant heterogeneity among samples $(Q[6]=$ 4.90, $p=0.557$ ). The results are presented as a forest plot in Figure 2.

\begin{tabular}{|c|c|c|}
\hline Lee et al., 2005 [19] & $\longmapsto$ & $0.19[-0.01,0.37]$ \\
\hline Mulert et al., 2002 [21] & $\frac{1}{1}$ & $0.31[-0.24,0.71]$ \\
\hline Lee et al., 2015 [12] & 1 & $0.33[0.02,0.58]$ \\
\hline Gallinat et al., 2000 [20] & & $0.39[0.03,0.66]$ \\
\hline Park et al., 2011 [13] & & $0.46[0.08,0.72]$ \\
\hline Juckel et al., 2007 [17] & & $0.47[0.03,0.76]$ \\
\hline Mulert et al., 2007 [18] & & $0.66[0.10,0.90]$ \\
\hline & & $0.33[0.20,0.45]$ \\
\hline & 0.0 & 1.0 \\
\hline
\end{tabular}

Back-transformed correlation coefficient

Fig. 2. Forest plot of the results.

RE model, random-effects model.

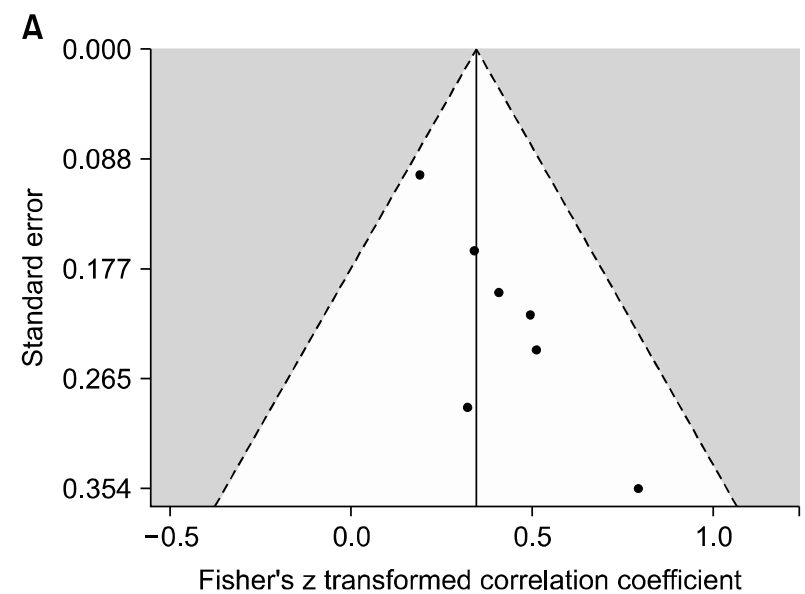

Given that depressive disorders and GAD involve different underlying mechanisms [22], we ran an additional analysis using only the studies that examined depressive disorders. A random-effects model of the LDAEP and SSRIs treatment response in patients with depression yielded a medium positive correlation $(r=0.31,95 \% \mathrm{Cl}$ [0.16, 0.44], $z=4.17, p<0.001,95 \%$ CR [0.14, 0.47], $Q[5]=4.20, p=0.521)$.

\section{Publication Bias}

Using visual inspection of funnel plot and Egger's regression test, publication bias can be detected by testing the asymmetry of funnel plot that shows studies with smaller sample size tended to have been published with larger effect sizes. Effect sizes appear asymmetrical on the funnel plot (Fig. 3A) and the Egger test results showed a significant asymmetry, indicating potential publication bias, $z=1.97, p=0.048$. To adjust the observed publication bias, we used the trim and fill method to impute potentially missing studies. The method imputed 4 studies on the left side of the funnel plot (Fig. 3B). With the imputed data, the overall effect size of the relationship between baseline LDAEP values and treatment response remained significant and was close to medium $(r=0.24,95 \% \mathrm{Cl}$ $[0.13,0.34], z=4.14, p<0.001,95 \%$ CR [0.13, 0.34]).

\section{DISCUSSION}

This study aims to quantify the overall relationship between pre-treatment LDAEP values and response to SSRIs in patients with depression and anxiety disorders across

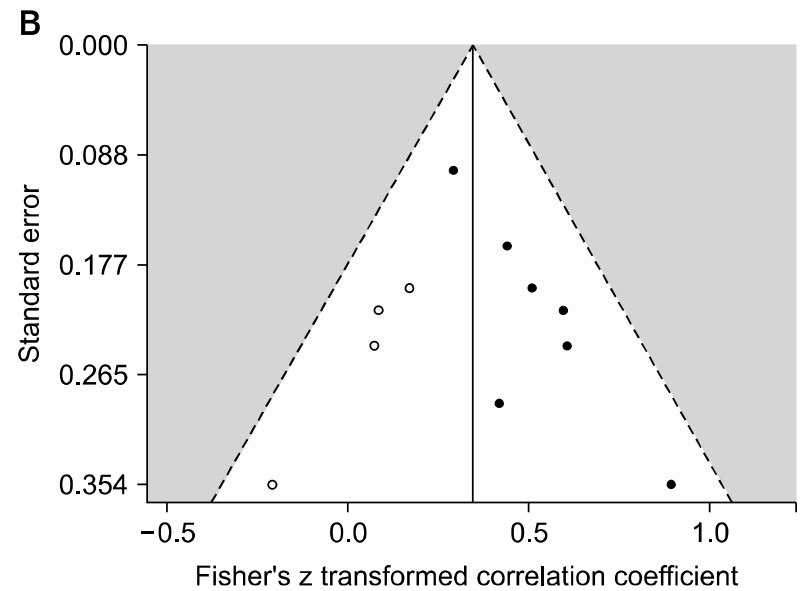

Fig. 3. Funnel plots of the results. (A) Funnel plot before adjustment. (B) Funnel plot with imputed studies. 
seven studies. Response to SSRIs were defined as reductions in clinical symptoms at the post-treatment. Using a meta-analysis, we found that pre-treatment LDAEP has a medium and positive relationship with treatment response to SSRIs. As predicted, patients with stronger pre-treatment LDAEP scores had greater reductions in clinical symptoms after SSRIs treatment.

Our results confirmed the argument of previous narrative reviews [8-11] that pre-treatment LDAEP has a significant utility as a biomarker to predict response to SSRIs treatment. Why does baseline LDAEP predict response to SSRIs? The most commonly cited explanation regards 5-HT function. Many studies claimed that LDAEP reflects 5-HT activity levels: stronger LADEP indicates the lower levels of $5-\mathrm{HT}$ function $[7,12,17]$. Based on this assumption, it might be possible that a person with lower baseline 5-HT levels may particularly benefit from SSRIs because the medication enhances 5-HT levels in the brain system. On the other hand, those with weaker baseline LDAEP values, which indicates relatively "normal" 5-HT levels, might not have enough room in their system to take advantage of additional 5-HT provided by SSRIs. This hypothesis is plausible; however, conflicting evidence exists on whether LDAEP sensitively and specifically measures 5-HT activities [8].

Another possible explanation is that LDAEP may reflect the functions of multiple neurotransmitters that are broadly related to psychiatric illnesses, such as dopamine, glutamate as well as $5-\mathrm{HT}[8,23,24]$. It might be possible that stronger baseline LDAEP indicates lower activity levels of or imbalance between those neurotransmitters. Individuals with stronger baseline LDAEP might more likely benefit from treatments targeting certain neurotransmitters, such as 5-HT using SSRIs, compared to those with weaker LDAEP. In addition, Lee et al. [25] found that stronger LDAEP predicts favorable response to repetitive transcranial magnetic stimulation (rTMS) in patients with depression. Although rTMS does not specifically target neurotransmitters, previous studies suggested its influence on various neurotransmitters through impacting circuitry, such as GABAergic [26], dopaminergic [27], 5-HT circuitry [28], and norepinephrine neurons [29], which is consistent with the idea that LDAEP may be related to multiple neurotransmitters. These results lead to a question on whether baseline LDAEP would predict a treatment response to non-biological treatments, such as psy- chotherapy, or its treatment prediction is specific to biological treatments, such as SSRIs and rTMS. Addressing this question in future research will broaden our understanding of the mechanism(s) as well as the utility of LDAEP.

Although underlying mechanism remains unclear, the current results of LDAEP significantly predicting response to SSRIs are compelling for its potential clinical utility. Literature on treatment selection highlighted that examining biomarkers might be an essential key to decipher the complexity of treatment response prediction [30]. Compared to other biomarker-assessments, such as neuroimaging measures, LDAEP is relatively easy to administer using an EEG, a non-invasive and cost-effective tool. Its ease of use makes LDAEP an attractive assessment measure that can be implemented as a part of routine medical procedures before administering medications. Further, two previous studies demonstrated that LDAEP can differentially predict response to NRI (i.e., reboxetine) and SSRIs $[17,18]$ : lower levels of pre-treatment LDAEP predicts favorable response to NRI, whereas SSRIs have an inverse relationship. This suggests the possibility of using LDAEP as a treatment selection biomarker. With more accumulated individual studies, a future meta-analysis is needed to compare LDAEP's predictability of treatment outcomes between SSRIs vs. non-SSRIs pharmacotherapies.

Despite the promising utility of LDAEP as a clinical biomarker, there exist a couple of critical issues. The first issue regards data acquisition and analysis. It is essential to standardize LDAEP assessment and analysis across individual studies to ensure the reliability and validity of LDAEP and its interpretations. In the method section, we presented the most commonly used method among the included studies. We strongly encourage researchers to consider this method when conducting a future LDAEP research. In addition, the operationalization of LDAEP is necessary to use LDAEP as a biomarker in clinical setting. A uniform cut-off score to represent strong or weak LDEAP would be ideal. However, it would require a large sample size to evaluate the effects of potential moderators (e.g., sex, age, ethnicity) on the uniform cut-off value. A more feasible next step may be to establish a base LDAEP score within each clinical setting/laboratory. For instance, one may assess a base LDAEP average score and variance, as well as moderators, with an adequate number of patients within a clinic. The clinic may define strong and week LDEAP values using one standard deviation below 
and above the base score. Those strong/weak LDAEP scores can be used to guide the prediction of outcomes to SSRIs in the clinic. This idea needs an empirical investigation for its clinical utility and practicality.

The current study has limitations. First, due to lacking previous studies on this topic, the current study has a small sample size. Because $Q$ test is sensitive to sample size [31], existing heterogeneity among effect sizes might have not been detected. Further, we could not test potential moderators due to the small sample size. Second, our paper addressed both depression and anxiety; however, only one included study examined GAD. Our results may not be generalized to other anxiety disorders. Future studies are needed to examine whether the significant predictability of LDAEP is found in other psychiatric disorders that use SSRIs as their first line treatments, including various anxiety disorders. Finally, many of the included studies came from a handful of research groups. This may have inflated the overall effect size and limited heterogeneity among effect sizes. More research from diverse laboratories is imperative to tackle this issue.

In conclusion, this study is the first meta-analysis to quantify the overall relationship between pre-treatment LDAEP values and treatment response to SSRIs for depression and GAD across individual studies. We found that stronger baseline LDAEP values predicted favorable response to SSRIs treatment across studies. The current study provides empirical evidence for the clinical utility of LDAEP as a biomarker for response prediction to SSRIs.

\section{Acknowledgments}

This work was partly supported by the Institute of Information \& communications Technology Planning \& Evaluation (IITP) grant funded by the Korea government (MSIT) (No. 2020-0-01376, Development of the multispeaker conversational speech recognition technology) and the Korea Science and Engineering Foundation (KOSEF), funded by the Korean government (NRF-2018R1A2A2A0 5018505)

\section{Conflicts of Interest}

No potential conflict of interest relevant to this article reported.

\section{Author Contributions}

Conceptualization: Seung-Hwan Lee, Data curation:
Sunkyung Yoon, Yourim Kim. Formal analysis: Sunkyung Yoon, Yourim Kim. Writing-original draft: Sunkyung Yoon, Yourim Kim. Writing - review \& editing: Seung-Hwan Lee. All authors contributed to and have approved the final manuscript.

\section{ORCID}

Sunkyung Yoon Yourim Kim https://orcid.org/0000-0002-2809-8877 https://orcid.org/0000-0003-0305-3709

\section{REFERENCES}

1. Lin SH, Lee LT, Yang YK. Serotonin and mental disorders: a concise review on molecular neuroimaging evidence. Clin Psychopharmacol Neurosci 2014;12:196-202.

2. Naughton M, Mulrooney JB, Leonard BE. A review of the role of serotonin receptors in psychiatric disorders. Hum Psychopharmacol 2000;15:397-415.

3. Vaswani M, Linda FK, Ramesh S. Role of selective serotonin reuptake inhibitors in psychiatric disorders: a comprehensive review. Prog Neuropsychopharmacol Biol Psychiatry 2003; 27:85-102.

4. Baldwin DS, Anderson IM, Nutt DJ, Allgulander C, Bandelow $\mathrm{B}$, den Boer JA, et al. Evidence-based pharmacological treatment of anxiety disorders, post-traumatic stress disorder and obsessive-compulsive disorder: a revision of the 2005 guidelines from the British Association for Psychopharmacology. J Psychopharmacol 2014;28:403-439.

5. Kennedy SH, Lam RW, Cohen NL, Ravindran AV. Clinical guidelines for the treatment of depressive disorders. IV. Medications and other biological treatments. Can J Psychiatry 2001;46 Suppl 1:38S-58S.

6. Charney DS, Buxbaum JD, Sklar P, Nestler EJ. Neurobiology of mental illness. 4th ed. Oxford:Oxford University Press; 2013.

7. Hegerl U, Gallinat J, Juckel G. Event-related potentials. Do they reflect central serotonergic neurotransmission and do they predict clinical response to serotonin agonists? J Affect Disord 2001;62:93-100.

8. O'Neill BV, Croft RJ, Nathan PJ. The loudness dependence of the auditory evoked potential (LDAEP) as an in vivo biomarker of central serotonergic function in humans: rationale, evaluation and review of findings. Hum Psychopharmacol 2008;23: 355-370.

9. Papakostas GI, Fava M, Thase ME. Treatment of SSRI-resistant depression: a meta-analysis comparing within-versus acrossclass switches. Biol Psychiatry 2008;63:699-704.

10. Perlman K, Benrimoh D, Israel S, Rollins $C$, Brown E, Tunteng $\mathrm{JF}$, et al. A systematic meta-review of predictors of antidepressant treatment outcome in major depressive disorder. J Affect Disord 2019;243:503-515. 
11. Pogarell O, Juckel G, Norra C, Leicht G, Karch S, Schaaff N, et al. Prediction of clinical response to antidepressants in patients with depression: neurophysiology in clinical practice. Clin EEG Neurosci 2007;38:74-77.

12. Lee BH, Park YM, Lee SH, Shim M. Prediction of long-term treatment response to selective serotonin reuptake inhibitors (SSRIs) using scalp and source loudness dependence of auditory evoked potentials (LDAEP) analysis in patients with major depressive disorder. Int J Mol Sci 2015;16:6251-6265.

13. Park YM, Kim DW, Kim S, Im CH, Lee SH. The loudness dependence of the auditory evoked potential (LDAEP) as a predictor of the response to escitalopram in patients with generalized anxiety disorder. Psychopharmacology (Berl) 2011;213: 625-632.

14. Landis JR, Koch GG. The measurement of observer agreement for categorical data. Biometrics 1977;33:159-174.

15. Cohen J. A power primer. Psychol Bull 1992;112:155-159.

16. Viechtbauer W. Conducting meta-analyses in $R$ with the metafor package. J Stat Softw 2010;36:1-48.

17. Juckel G, Pogarell $\mathrm{O}$, Augustin H, Mulert C, Müller-Siecheneder F, Frodl T, et al. Differential prediction of first clinical response to serotonergic and noradrenergic antidepressants using the loudness dependence of auditory evoked potentials in patients with major depressive disorder. J Clin Psychiatry 2007; 68:1206-1212.

18. Mulert C, Juckel G, Brunnmeier M, Karch S, Leicht G, Mergl R, et al. Prediction of treatment response in major depression: integration of concepts. J Affect Disord 2007;98:215-225.

19. Lee TW, Yu YW, Chen TJ, Tsai SJ. Loudness dependence of the auditory evoked potential and response to antidepressants in Chinese patients with major depression. J Psychiatry Neurosci 2005;30:202-205.

20. Gallinat J, Bottlender R, Juckel G, Munke-Puchner A, Stotz G, Kuss $\mathrm{HJ}$, et al. The loudness dependency of the auditory evoked N1/P2-component as a predictor of the acute SSRI response in depression. Psychopharmacology (Berl) 2000;148: 404-411.

21. Mulert C, Juckel G, Augustin H, Hegerl U. Comparison between the analysis of the loudness dependency of the auditory N1/P2 component with LORETA and dipole source analysis in the prediction of treatment response to the selective serotonin reuptake inhibitor citalopram in major depression. Clin Neurophysiol. 2002;113:1566-1572.

22. Mennin DS, Heimberg RG, Fresco DM, Ritter MR. Is generalized anxiety disorder an anxiety or mood disorder? Considering multiple factors as we ponder the fate of GAD. Depress Anxiety 2008;25:289-299.

23. Hitz K, Heekeren K, Obermann C, Huber T, Juckel G, Kawohl W. Examination of the effect of acute levodopa administration on the loudness dependence of auditory evoked potentials (LDAEP) in humans. Psychopharmacology (Berl) 2012;221: 389-396.

24. O'Neill BV, Croft RJ, Leung S, Oliver C, Phan KL, Nathan PJ. High-dose glycine inhibits the loudness dependence of the auditory evoked potential (LDAEP) in healthy humans. Psychopharmacology (Berl) 2007; 195:85-93.

25. Lee $\mathrm{S}$, Jang KI, Chae JH. Association of the loudness dependence of auditory evoked potentials with clinical changes to repetitive transcranial magnetic stimulation in patients with depression. J Affect Disord 2018;238:451-457.

26. Trippe J, Mix A, Aydin-Abidin S, Funke K, Benali A. $\theta$ burst and conventional low-frequency rTMS differentially affect GABAergic neurotransmission in the rat cortex. Exp Brain Res 2009; 199:411-421.

27. Pogarell O, Koch W, Pöpperl G, Tatsch K, Jakob F, Mulert C, et al. Acute prefrontal rTMS increases striatal dopamine to a similar degree as D-amphetamine. Psychiatry Res 2007;156: 251-255.

28. Baeken C, De Raedt R, Bossuyt A, Van Hove C, Mertens J, Dobbeleir A, et al. The impact of HF-rTMS treatment on serotonin(2A) receptors in unipolar melancholic depression. Brain Stimul 2011;4:104-111.

29. Moisset X, de Andrade DC, Bouhassira D. From pulses to pain relief: an update on the mechanisms of rTMS-induced analgesic effects. Eur J Pain 2016;20:689-700.

30. Cohen ZD, DeRubeis RJ. Treatment selection in depression. Annu Rev Clin Psychol 2018;14:209-236.

31. Huedo-Medina TB, Sánchez-Meca J, Marín-Martínez F, Botella J. Assessing heterogeneity in meta-analysis: Q statistic or $I^{2}$ index? Psychol Methods 2006;11:193-206. 